\title{
Clinical pharmacokinetics of encapsulated oral 9-aminocamptothecin in plasma and saliva
}

Objective: To study the pharmacokinetics and pharmacodynamics of the novel topoisomerase I inhibitor and antitumor agent 9-amino-20(S)-camptothecin in patients with solid tumors after repeated oral administration.

Methods: Thirty-two patients with cancer received oral 9-aminocamptothecin formulated in capsules with polyethylene glycol-1000 as excipient at doses that ranged from 0.25 to $1.5 \mathrm{mg} / \mathrm{m}^{2} /$ day. Serial plasma and saliva samples were obtained on days 1 and 6 or days 1 and 8 of the first cycle and analyzed for the lactone and carboxylate forms of 9 -aminocamptothecin by HPLC.

Results: 9-Aminocamptothecin showed linear and dose-independent pharmacokinetics, with extremely small intrapatient kinetic variability (coefficient of variation: $<10 \%$ ). However, interpatient variability in plasma pharmacokinetics was large (coefficient of variation: 99\%). The relative extent of lactone to carboxylate interconversion was large $(>90 \%)$ and predictable from individual pretreatment serum albumin values $(P=.0099)$. The 9 -aminocamptothecin concentration ratio in plasma and saliva was strongly patient dependent, and highly variable around a mean value of $<0.8$, suggesting that saliva is an unreliable matrix for kinetic monitoring. The area under the curve of the lactone form of 9-aminocamptothecin was significantly correlated with the dose-limiting hematologic toxicity $(P<.001)$.

Conclusion: Our data indicate that the large interindividual pharmacodynamic variability in response to 9aminocamptothecin is caused mainly by a variability in kinetic characteristics, suggesting that a kineticdynamic guided study design is warranted in future clinical investigations. (Clin Pharmacol Ther 1999;65:491-9.)

\author{
Maja J. A. de Jonge, MD, Jaap Verweij, MD, PhD, Walter J. Loos, BSc, \\ Brian K. Dallaire, PharmD, and Alex Sparreboom, PhD \\ Rotterdam, The Netherlands, and San Diego, Calif
}

In the early 1970 s, camptothecin, a plant alkaloid extract from the bark and wood of the Chinese tree Camptotheca acuminata, was shown to possess antineoplastic activity. ${ }^{1}$ Analogs of camptothecin belong to a family of anticancer agents with a unique mechanism of action that is based on reversible inhibition of DNA topoisomerase I. ${ }^{2,3}$

From the Department of Medical Oncology, Rotterdam Cancer Institute (Daniel den Hoed Kliniek) and University Hospital Rotterdam, Rotterdam, and IDEC Pharmaceuticals Corporation, San Diego.

Received for publication July 30, 1998; accepted Jan 23, 1999.

Reprint requests: Maja J. A. de Jonge, MD, Department of Medical Oncology, Rotterdam Cancer Institute (Dr Daniel den Hoed Kliniek) and University Hospital Rotterdam, Groene Hilledijk 301, 3075 EA Rotterdam, The Netherlands.

Copyright () 1999 by Mosby, Inc.

$0009-9236 / 99 / \$ 8.00+0 \quad \mathbf{1 3 / 1 / 9 7 4 2 8}$
Despite the cytotoxicity of the compound, further development was halted because of a number of severe and unpredictable side effects observed in early clinical trials. ${ }^{4-6}$ The subsequent search for less toxic analogs of camptothecin resulted in the discovery of irinotecan, topotecan, 9-amino-20(S)-camptothecin, 9nitrocamptothecin, DX-8951f, and GI147211. The first two-irinotecan and topotecan-were recently registered for the treatment of colorectal and ovarian cancer, respectively.

In aqueous solutions, camptothecins are unstable and undergo a rapid, pH-dependent, nonenzymatic hydrolysis of the terminal lactone ring to form the more water-soluble, ring-opened carboxylate form (see Structure). ${ }^{7}$ The presence of the intact terminal lactone ring is thought to be essential for the topoisomerase I inhibition. ${ }^{8}$ The closed lactone ring predominates at acidic $\mathrm{pH}$, whereas in human plasma the equilibrium 


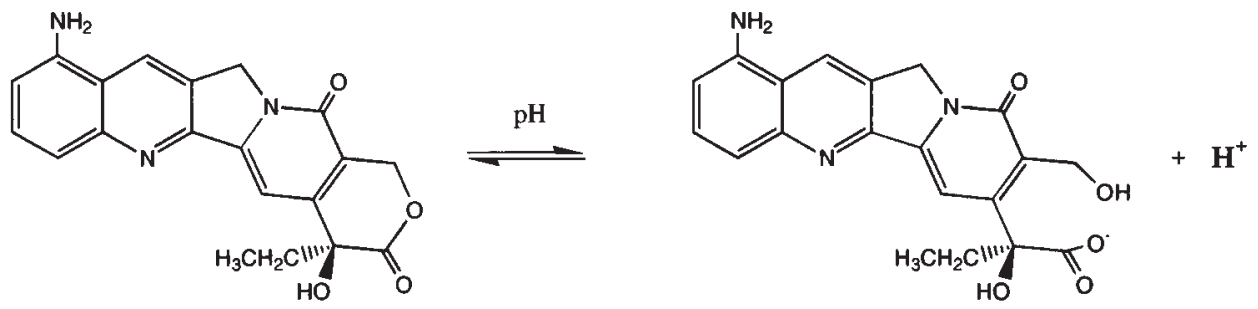

Chemical structure and $\mathrm{pH}$-dependent interconversion of 9-amino-20(S)-camptothecin lactone and 9-amino-20(S)-camptothecin carboxylate.

between these 2 species greatly favors formation of the carboxylate form, partly because of the physiologic $\mathrm{pH}$ and the preferentially binding of this form to albu$\min .9,10$ The ratio of the lactone form to the total drug concentration at steady state in plasma is different for each camptothecin analog, which might have important pharmacokinetic and pharmacodynamic implications.

In preclinical studies 9-aminocamptothecin demonstrated activity against human colon, breast, prostate, nonsmall cell lung cancer, and melanoma xenografts. ${ }^{11-14}$ Preclinical in vivo data suggested that duration of exposure to 9 -aminocamptothecin lactone above a certain threshold concentration $(10 \mathrm{nmol} / \mathrm{L})$ and frequency of administration were essential for antitumor activity. ${ }^{15,16}$

Based on its preclinical activity, 9-aminocamptothecin appeared to merit evaluation as an antineoplastic agent. To mimic the preclinical studies, initial phase I studies that used the intravenous formulation of 9aminocamptothecin focused on schedules with prolonged infusion duration of 24 to 120 hours $^{17-21}$ or a continuous infusion for 21 days every 4 weeks. ${ }^{22}$ Pharmacokinetic data obtained during these studies showed marked interpatient variability. Steady-state plasma concentrations of 9-aminocamptothecin lactone greater than $10 \mathrm{nmol} / \mathrm{L}$ were achieved only in the phase I study of the 24-hour infusion of 9-aminocamptothecin at the dose recommended for further phase II studies (ie, 1.65 $\mathrm{mg} / \mathrm{m}^{2}$ ). When 9 -aminocamptothecin was administered as a 72-hour infusion once every 2 or 3 weeks, the maximal tolerated dose was 35 to $54.2 \mu \mathrm{g} / \mathrm{m}^{2} / \mathrm{h}$. The doselimiting toxicity consisted of neutropenia in combination with thrombocytopenia and correlated to the steadystate 9-aminocamptothecin lactone concentration.

We recently reported that 9 -aminocamptothecin demonstrated rapid absorption in humans after oral delivery, with an overall bioavailability of approximately $50 \% .{ }^{23}$ In the present report, we present a comprehensive analysis of the plasma pharmacokinetics of the lactone and carboxylate forms of 9-aminocamptothecin in patients with cancer receiving the drug orally over a wide range of dose levels, with special focus on pharmacokinetic-pharmacodynamic characteristics. To assess the clinical usefulness of salivary monitoring of 9-aminocamptothecin for kinetic modeling, paired plasma and coinciding unstimulated saliva samples were collected in a limited number of patients.

\section{PATIENTS AND METHODS}

Patient population. Pharmacokinetic curves were obtained from patients who participated in an oral bioavailability study of 9-aminocamptothecin and/or in a phase I trial of oral 9-aminocamptothecin administered daily for 7 to 14 consecutive days every 3 weeks. Treatment plans and detailed clinical profiles have been documented elsewhere. ${ }^{23}$ Eligibility criteria included a histologically or cytologically confirmed diagnosis of a solid malignant tumor not amenable to established forms of treatment. All patients had adequate hematopoietic function (absolute peripheral granocyte count $\geq 2.0 \times 10^{9} / \mathrm{L}$ and platelet count $\geq 100 \times 10^{9} / \mathrm{L}$ ), hepatic function (bilirubin within normal limits, and serum aspartate aminotransferase, alanine aminotransferase, and alkaline phosphatase $\leq 2.5$ times the normal limit), and renal function (serum creatinine $<133$ $\mu \mathrm{mol} / \mathrm{L})$. Other eligibility criteria included the following: age between 18 and 75 years, Eastern Cooperative Oncology Group performance status $\leq 2$, estimated life expectancy $\geq 12$ weeks, no previous anticancer therapy for at least 4 weeks ( 6 weeks for nitrosourea or mitomycin), and no previous therapy with other camptothecins or intensive ablative regimens. Specific exclusion criteria included long-term treatment with corticosteroids and significant gastrointestinal dysfunction that could alter absorption or motility. Concomitant administration of $\mathrm{H}_{2}$-antagonists, antacids, protonpump inhibitors, and nonsteroidal anti-inflammatory drugs were avoided. All patients gave written informed consent before study entry.

Treatment plan and dose escalation. The oral formulation of 9-aminocamptothecin was supplied by Pharma- 
cia \& Upjohn (Nerviano, Italy) as hard gelatin capsules that contained $0.10,0.25$, or $1 \mathrm{mg}$ of the active drug and polyethylene glycol-1000 (PEG-1000) as excipient. ${ }^{23}$ The capsules were stored at room temperature.

A detailed description of the preparation and rationale for composition of the formulation has been described previously. ${ }^{23}$ Patients received 9-aminocamptothecin orally with 150 to $200 \mathrm{~mL}$ water at dose levels of $0.25,0.40,0.60,0.84,1.0$, or $1.1 \mathrm{mg} / \mathrm{m}^{2} /$ day for 7 or 14 consecutive days or as a single dose of 1.5 $\mathrm{mg} / \mathrm{m}^{2}$. Intrapatient dose escalation in the phase I trial was not permitted. Weekly evaluation of the patients included a clinical history, physical examination, toxicity assessment according to common toxicity criteria, and serum chemistry. A complete blood cell count with differentiation was determined twice weekly.

Sample collection and drug analysis. Serial blood samples were collected in heparinized tubes from an indwelling venous catheter at $0,0.33,0.67,1,1.5,2,3$, $5,7.5,11$, and 24 hours after administration on days 1 and 6 or days 1 and 8 of the first treatment course. In a limited number of patients, unstimulated saliva samples were obtained at coinciding time points. In 1 patient with a malignant pleural effusion, additional pleural fluid sampling was performed to assess the influence of the pleural effusion on the plasma pharmacokinetics. Immediately after sampling, aliquots of plasma (separated at $4^{\circ} \mathrm{C}$ ), pleural fluid, and saliva were frozen at $-80^{\circ} \mathrm{C}$, and analyzed later for 9-aminocamptothecin lactone and total 9-aminocamptothecin (ie, lactone plus carboxylate) with use of a validated reversedphase HPLC method as described previously. ${ }^{24}$ Drug concentrations in patient plasma samples were calculated with use of interpolation of the corresponding regression analysis. Specimens with drug levels that exceeded the upper range of the calibration curve were reanalyzed after appropriate dilution with drug-free plasma. Saliva and pleural fluid samples were diluted 4-fold in drug-free plasma and analyzed with use of the same analytical assay, with minor modifications. The lower limits of detection of the assays were $0.05 \mathrm{ng} / \mathrm{mL}$ in plasma and $0.4 \mathrm{ng} / \mathrm{mL}$ in pleural fluid and saliva.

Data analysis. Plasma concentration data were analyzed with both noncompartmental and compartmental analysis by use of the Siphar software package (version 4.0, Simed, Créteil, France). The model-independent pharmacokinetic parameters included the maximum plasma concentration and the time to reach maximum plasma concentration. Initial parameter estimates were obtained with use of an automated peeling algorithm based on the Powell method to 3 compartments, which yielded the best statistical fit as determined by the
Table I. Patient characteristics

\begin{tabular}{lc}
\hline No. of patients entered & 32 \\
No. of patients assessable for toxicity & 30 \\
Age (yr) & \\
Median & 59 \\
Range & $29-74$ \\
Sex (n) & \\
Female & 14 \\
Male & 16 \\
Performance status & \\
Median & 1 \\
Range & $0-2$ \\
Tumor type (n) & \\
Colorectal & 14 \\
Ovarian & 5 \\
Sarcoma & 2 \\
Mesothelioma & 2 \\
Lung (non-small cell) & 2 \\
Miscellaneous & 5 \\
Previous treatment (n) & \\
Chemotherapy & 16 \\
Radiation & 2 \\
Chemotherapy and radiation & 2 \\
None & \\
\hline
\end{tabular}

$\mathrm{n}$, Number of patients.

Akaike information criterion and the $\mathrm{F}$ test. The area under the plasma concentration-time curve (AUC) values were calculated on the basis of the best-fitted curve, as were the disposition half-lives. The apparent absorption rate constant was obtained through numerical point-area deconvolution. Relationships between the AUC and pharmacodynamic outcome were evaluated with (log-)linear and (non-)sigmoidal maximum effect modeling by use of Siphar and NCSS version 5.0 (Dr Jerry Hintze, East Kayesville, Utah). Within individual patients, myelosuppression was described as the continuous variable, consisting of the percentage decrease in white blood cell count, absolute neutrophil count, and platelet count. The relative hematologic toxicity was defined as follows:

$\%$ Decrease $=$

(Pretherapy value - Nadir value)/(Pretherapy value) $\cdot 100$

Only the first course of each patient was taken into consideration to avoid potentially confounding bias caused by cumulative toxicity. All data were fitted to a sigmoidal maximum effect $\left(\mathrm{E}_{\max }\right)$ model based on the modified Hill equation, as follows:

$$
\mathrm{E}=\mathrm{E}_{0}+\mathrm{E}_{\max } \cdot\left[(\mathrm{KP} \gamma) /\left(\mathrm{KP}^{\gamma}+\mathrm{KP}_{50} \gamma\right)\right]
$$

In this equation, $\mathrm{E}_{0}$ is the minimum reduction possible (fixed at a value of 0 ), $E_{\max }$ is the maximum response (fixed at 100), KP is the pharmacokinetic parameter of 


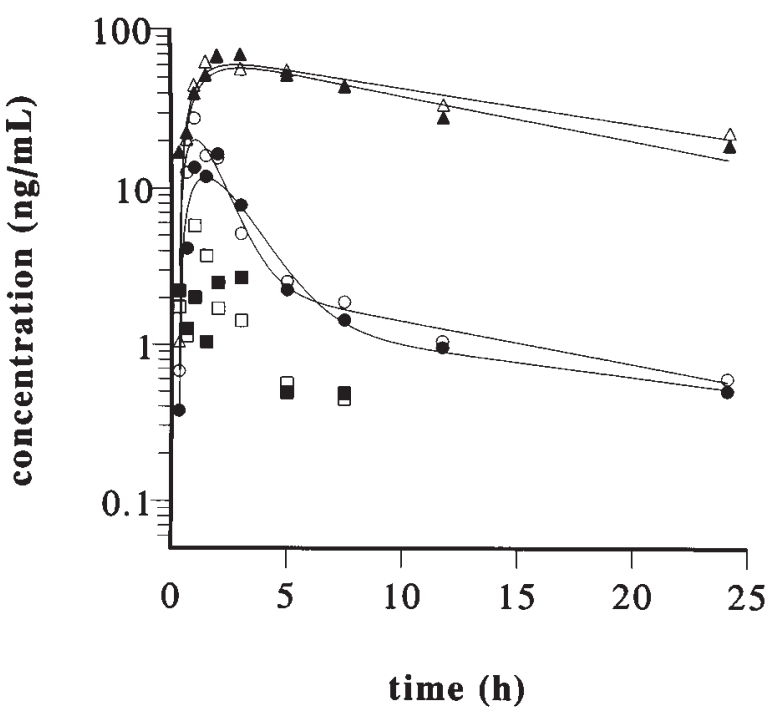

Figure 1. Representative concentration-time profiles of 9aminocamptothecin lactone (circles) and total 9-aminocamptothecin (triangles) in plasma and in saliva (squares) measured on day 1 (open symbols) and day 8 (solid symbols) of the first treatment course in a single patient after oral administration of 9-aminocamptothecin at a dose level of 0.84 $\mathrm{mg} / \mathrm{m}^{2} /$ day for 14 days. All pharmacokinetic curves were fitted to a triexponential equation, assuming a 3-compartment modal for the distribution and elimination of the drug.

interest, $\mathrm{KP}_{50}$ is the value of the pharmacokinetic parameter predicted to result in half of the maximum response, and $\gamma$ is the Hill constant, which describes the sigmoidicity of the curve. Models were evaluated for goodness of fit by minimization of sums of the squared residuals and by reduction of the estimated coefficient of variation for fitted parameters. Significance of the relationships were assessed by construction of contingency tables with subsequent $\chi^{2}$ analysis.

\section{RESULTS}

Demographic characteristics of all 32 patients who had blood sampling for pharmacokinetic analysis are shown in Table I. Ten patients completed the oral bioavailability study and subsequently participated in the phase I and pharmacokinetic study of oral 9aminocamptothecin. Pharmacokinetic data were therefore obtained in 42 courses. Plasma pharmacokinetics could not be determined in 1 course on day 1 and in 2 courses on day 8 as a result of limited sample availability or significant chromatographic interference in the drug assay by an unknown compound. Twenty-seven patients were assessable for pharmacodynamic analysis and 30 patients were assessable for toxicity.
The plasma concentration-time profiles of 9aminocamptothecin lactone and 9-aminocamptothecin carboxylate were similar for all patients studied, with representative examples shown in Figure 1. The pharmacokinetics of both species of 9-aminocamptothecin could be best described with a 3-compartment model. The kinetic parameters obtained by means of this model are presented in Tables II and III. The absorption of 9aminocamptothecin lactone after oral administration was rapid, with a lag-time of $0.29 \pm 0.07$ hour (mean $\pm \mathrm{SD} ; \mathrm{n}=41)$, maximum peak drug levels at $0.99 \pm$ 0.12 hour, and a mean absorption rate constant of 3.03 $\pm 0.90 \mathrm{~h}^{-1}$. In the first plasma samples the ring-opened carboxylate form of 9-aminocamptothecin was already detectable. Maximal plasma concentrations of 9aminocamptothecin carboxylate were reached at 2.41 \pm 0.64 hours after administration. The 9 -aminocamptothecin carboxylate eventually accounted for $91.1 \% \pm$ $2.11 \%$ of 9 -aminocamptothecin total drug concentrations, indicating a clear predominant conversion of lactone to carboxylate. Elimination of 9-aminocamptothecin from the central plasma compartment was characterized by a decay in an apparent triexponential manner based on conventional compartment modeling with use of weighed least-squares analysis with a weighting factor of $1 / Y$. The mean values for the linear segments of 9 -aminocamptothecin lactone were $t_{1 / 2} \alpha$ : 0.26 hour (range, 0.13 to 0.38 hour), $\mathrm{t}_{1 / 2} \beta$ : 0.78 hour (range, 0.54 to 1.13 hours) and $t_{1} / 2: 7.47$ hours (range, 3.66 to 12.6 hours). No significant quantitative differences were observed between the decay kinetics of 9aminocamptothecin lactone and 9-aminocamptothecin carboxylate.

The kinetic data and recorded AUC values for the following days of administration were similar to those achieved the first day in the same patient (Tables II and III). Therefore the resulting intrapatient variability in AUC and peak drug levels, expressed as the coefficient of variation, was extremely small and averaged $8.67 \%$ for 9 -aminocamptothecin lactone and $10.9 \%$ for 9 aminocamptothecin carboxylate. However, the interpatient variability in the observed pharmacokinetics was large, with coefficients of variation in AUC values as high as $89.5 \%$ for 9 -aminocamptothecin lactone and $99.0 \%$ for 9 -aminocamptothecin carboxylate.

Over the total dose range studied, 9-aminocamptothecin lactone and total 9-aminocamptothecin showed linear and dose-independent pharmacokinetics (Figure $2, A$ and $B$, respectively). No significant relationship was observed between the AUC values of total 9-aminocamptothecin and that of the pharmacologically active species, 9-aminocamptothecin lactone (data not shown). The 
Table II. Model-dependent pharmacokinetics of 9-AC lactone after oral administration of 9-AC

\begin{tabular}{|c|c|c|c|c|c|c|c|}
\hline & \multicolumn{7}{|c|}{ Dose $\left(m g / m^{2} /\right.$ day $)$} \\
\hline & 0.25 & 0.40 & 0.60 & 0.84 & 1.0 & 1.1 & 1.5 \\
\hline $\mathrm{n}$ & 6 & 3 & 4 & 6 & 7 & 3 & 12 \\
\hline $\mathrm{k}_{\mathrm{a}}(\mathrm{L} / \mathrm{h})$ & ND & ND & ND & ND & ND & ND & $3.03 \pm 0.90$ \\
\hline $\mathrm{t}_{\mathrm{lag}}(\mathrm{h})$ & $0.33 \pm 0.13$ & $0.32 \pm 0.05$ & $0.38 \pm 0.14$ & $0.28 \pm 0.05$ & $0.29 \pm 0.05$ & $0.17 \pm 0.13$ & $0.24 \pm 0.13$ \\
\hline $\mathrm{t}_{\max }(\mathrm{h})$ & $1.06 \pm 0.35$ & $0.79 \pm 0.15$ & $0.94 \pm 0.11$ & $0.98 \pm 0.27$ & $0.99 \pm 0.29$ & $1.00 \pm 0.01$ & $1.17 \pm 0.33$ \\
\hline $\mathrm{C}_{\max }(\mathrm{ng} / \mathrm{mL})$ & $2.09 \pm 0.82$ & $7.94 \pm 7.39$ & $3.43 \pm 0.60$ & $4.61 \pm 1.51$ & $11.0 \pm 7.46$ & $12.2 \pm 6.95$ & $6.82 \pm 2.98$ \\
\hline $\mathrm{t}_{1} / 2 \alpha(\mathrm{h})$ & $0.25 \pm 0.20$ & $0.13 \pm 0.11$ & $0.23 \pm 0.07$ & $0.24 \pm 0.11$ & $0.24 \pm 0.09$ & $0.36 \pm 0.07$ & $0.38 \pm 0.21$ \\
\hline $\mathrm{t}_{1 / 2} / \beta(h)$ & $0.68 \pm 0.40$ & $0.82 \pm 0.33$ & $0.54 \pm 0.20$ & $0.73 \pm 0.33$ & $0.76 \pm 0.51$ & $0.82 \pm 0.16$ & $1.13 \pm 0.59$ \\
\hline $\mathrm{t}_{1 / 2} \gamma(\mathrm{h})$ & $5.22 \pm 4.22$ & $8.65 \pm 1.25$ & $3.66 \pm 0.55$ & $10.3 \pm 6.77$ & $5.10 \pm 2.68$ & $6.76 \pm 2.26$ & $12.6 \pm 4.20$ \\
\hline $\begin{array}{l}\text { AUC }(0-\infty) \text {, day } 1 \\
(\mathrm{ng} \cdot \mathrm{h} / \mathrm{mL})\end{array}$ & $6.88 \pm 4.76$ & $16.2 \pm 14.5$ & $8.15 \pm 0.76$ & $12.9 \pm 3.97$ & $31.2 \pm 19.1$ & $49.3 \pm 29.7$ & $31.9 \pm 14.3$ \\
\hline $\begin{array}{c}\text { AUC }(0-\infty) \text {, day } 8 \\
(\mathrm{ng} \cdot \mathrm{h} / \mathrm{mL})\end{array}$ & $9.48 \pm 3.85$ & $17.4 \pm 15.5$ & $8.69 \pm 0.70$ & $11.7 \pm 2.98$ & $31.2 \pm 27.0$ & $48.5 \pm 23.8$ & ND \\
\hline $\begin{array}{l}\text { Intrapatient } \\
\quad \% \mathrm{CV} \text { of AUC }\end{array}$ & 7.31 & 7.31 & 4.44 & 9.74 & 4.96 & 6.48 & ND \\
\hline $\begin{array}{l}\text { Interpatient } \\
\quad \% \mathrm{CV} \text { of AUC }\end{array}$ & 69.2 & 89.5 & 89.2 & 30.7 & 61.1 & 60.3 & 44.8 \\
\hline $\begin{array}{l}\text { Percent of total } \\
\text { 9-AC AUC }\end{array}$ & $7.18 \pm 2.33$ & $12.9 \pm 4.20$ & $8.76 \pm 3.49$ & $6.98 \pm 1.72$ & $10.2 \pm 4.94$ & $8.67 \pm 3.22$ & $7.30 \pm 3.22$ \\
\hline
\end{tabular}

$\mathrm{n}$, Number of patients; $\mathrm{k}_{\mathrm{a}}$, absorption rate constant; $\mathrm{t}_{\mathrm{lag}}$, lag-time; $\mathrm{t}_{\max }$, time to peak plasma levels; $\mathrm{C}_{\max }$, maximum plasma concentrations; $\mathrm{t}_{\mathrm{t} / 2}(\mathrm{i})$, half-life of the $\mathrm{i}^{\text {th }}$ disposition phase; AUC, area under the plasma concentration versus time curve; CV, coefficient of variation; 9-AC, 9-amino-20(S)-camptothecin.

Table III. Model-dependent pharmacokinetics of 9-AC carboxylate after oral administration of 9-AC

\begin{tabular}{|c|c|c|c|c|c|c|c|}
\hline & \multicolumn{7}{|c|}{ Dose $\left(m g / m^{2} /\right.$ day $)$} \\
\hline & 0.25 & 0.40 & 0.60 & 0.84 & 1.0 & 1.1 & 1.5 \\
\hline $\mathrm{n}$ & 6 & 3 & 4 & 6 & 7 & 3 & 12 \\
\hline $\mathrm{t}_{\mathrm{lag}}(\mathrm{h})$ & $0.41 \pm 0.20$ & $0.34 \pm 0.04$ & $0.39 \pm 0.13$ & $0.31 \pm 0.02$ & $0.35 \pm 0.15$ & $0.34 \pm 0.06$ & $0.31 \pm 0.11$ \\
\hline $\mathrm{t}_{\max }(\mathrm{h})$ & $2.60 \pm 1.19$ & $2.20 \pm 0.60$ & $1.63 \pm 0.23$ & $2.01 \pm 0.49$ & $2.36 \pm 1.19$ & $3.67 \pm 0.94$ & $2.39 \pm 1.05$ \\
\hline $\mathrm{C}_{\max }(\mathrm{ng} / \mathrm{mL})$ & $9.43 \pm 3.50$ & $11.8 \pm 5.76$ & $14.5 \pm 6.93$ & $15.2 \pm 4.88$ & $25.5 \pm 12.7$ & $41.8 \pm 19.6$ & $35.4 \pm 19.2$ \\
\hline $\mathrm{t}_{1} / 2 \mathrm{~h}(\mathrm{~h})$ & $0.55 \pm 0.50$ & $0.40 \pm 0.30$ & $0.20 \pm 0.20$ & $0.18 \pm 0.26$ & $0.24 \pm 0.38$ & $0.96 \pm 0.18$ & $1.04 \pm 0.78$ \\
\hline$t_{1} / 2 \beta(h)$ & $1.78 \pm 0.67$ & $1.12 \pm 0.44$ & $1.00 \pm 0.69$ & $1.07 \pm 0.55$ & $0.78 \pm 0.51$ & $4.26 \pm 1.60$ & $2.83 \pm 1.96$ \\
\hline $\mathrm{t}_{1 / 2} / \mathrm{\gamma}(\mathrm{h})$ & $7.50 \pm 3.96$ & $6.59 \pm 2.82$ & $4.98 \pm 1.74$ & $9.61 \pm 3.73$ & $8.62 \pm 3.57$ & $10.1 \pm 2.29$ & $12.4 \pm 6.48$ \\
\hline $\begin{array}{l}\text { AUC }(0-\infty) \text {, day } 1 \\
(\mathrm{ng} \cdot \mathrm{h} / \mathrm{mL})\end{array}$ & $90.8 \pm 48.2$ & $89.0 \pm 49.4$ & $102 \pm 45.1$ & $174 \pm 39.4$ & $357 \pm 265$ & $578 \pm 311$ & $510 \pm 354$ \\
\hline $\begin{array}{c}\text { AUC }(0-\infty) \text {, day } 8 \\
(\mathrm{ng} \cdot \mathrm{h} / \mathrm{mL})\end{array}$ & $177 \pm 127$ & $137 \pm 105$ & $83.9 \pm 34.1$ & $162 \pm 36.3$ & $315 \pm 327$ & $592 \pm 304$ & ND \\
\hline $\begin{array}{l}\text { Intrapatient } \\
\% \mathrm{CV} \text { of AUC }\end{array}$ & 13.9 & 27.7 & 1.63 & 8.52 & 6.49 & 10.5 & ND \\
\hline $\begin{array}{l}\text { Interpatient } \\
\quad \% \mathrm{CV} \text { of } \mathrm{AUC}\end{array}$ & 53.1 & 55.5 & 44.3 & 22.6 & 99.0 & 53.9 & 69.4 \\
\hline $\begin{array}{l}\text { Percent of total } \\
\text { 9-AC AUC }\end{array}$ & $92.8 \pm 2.33$ & $87.1 \pm 4.20$ & $91.2 \pm 3.49$ & $93.0 \pm 1.72$ & $89.8 \pm 4.94$ & $91.3 \pm 3.22$ & $92.7 \pm 4.15$ \\
\hline
\end{tabular}

$n$, Number of patients; $t_{\text {lag }}$, lag-time; $t_{\max }$, time to peak plasma levels; $\mathrm{C}_{\max }$, maximum plasma concentrations; $\mathrm{t}_{1 / 2}(\mathrm{i})$, half-life of the $\mathrm{i}^{\text {th }}$ disposition phase; AUC, area under the plasma concentration versus time curve; CV, coefficient of variation; 9-AC, 9-amino-20(S)-camptothecin.

interpatient variation in the equilibrium ratio of 9aminocamptothecin lactone and 9-aminocamptothecin carboxylate could be explained in part by individual differences in pretreatment serum albumin levels, for which a significant correlation with the AUC ratio of 9- aminocamptothecin lactone and 9-aminocamptothecin carboxylate could be shown $(r=0.471, P=.0099)$. This finding clearly indicates that separate monitoring of 9aminocamptothecin lactone and 9-aminocamptothecin carboxylate concentrations is mandatory to relate drug 


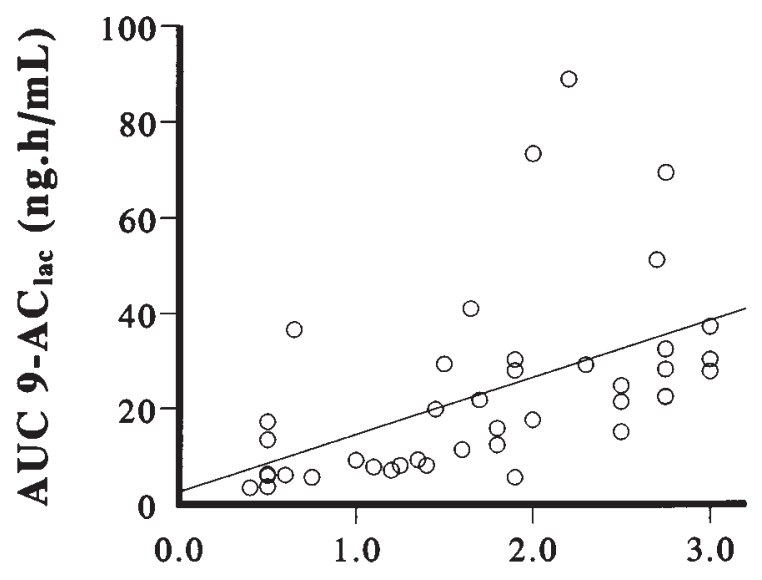

A dose (mg)

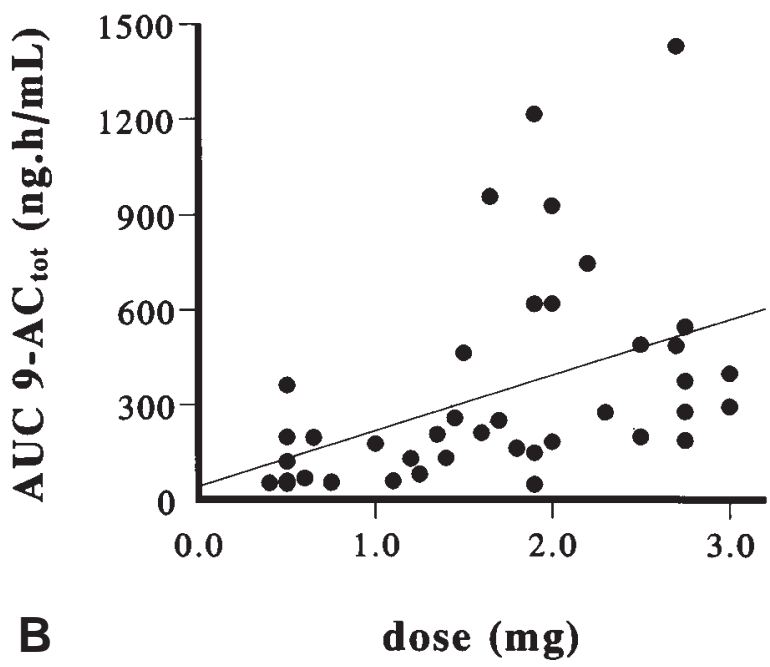

Figure 2. Absolute dose of 9-aminocamptothecin plotted versus area under the plasma concentration-time curve (AUC) of 9-aminocamptothecin lactone $\left(9-\mathrm{AC}_{\mathrm{lac}} ; \mathbf{A}\right)$ and total 9-aminocamptothecin $\left(9-\mathrm{AC}_{\text {tot }} ; \mathbf{B}\right)$.

levels to pharmacodynamic outcome in patients treated with oral 9-aminocamptothecin.

Salivary drug monitoring was evaluated as an option for determination of the AUC of 9-aminocamptothecin lactone. The 9-aminocamptothecin lactone concentration ratio in plasma and unstimulated saliva was strongly patient-dependent and highly variable around a mean value of $\sim 1.4$, suggesting that saliva is an unreliable matrix for pharmacokinetic analysis of this drug (Figure 1). To determine the impact of a pleural effusion on the pharmacokinetics of 9-aminocamptothecin, plasma and pleural effusion samples were obtained for drug analysis in a single patient with a malignant pleural effusion. The mean pleura versus plasma concentration ratio of 9-aminocamptothecin lactone was $4.95 \% \pm 2.32$ (mean $\pm \mathrm{SD}$; range, $0.7 \%$ to $6.8 \%$ ). These data indicate that pleural effusion does not constitute a major pharmacokinetic compartment for this drug.

The pharmacokinetic data obtained from 27 patients were plotted against the percentage decrease in white blood cell count, platelet count, and absolute neutrophil count at nadir relative to the pretreatment value. Four different models, based on linear, log-linear, $\mathrm{E}_{\max }$, and sigmoidal $\mathrm{E}_{\max }$ fitting, were compared for their ability to describe the data. With use of sigmoidal $\mathrm{E}_{\max }$ modeling of the pharmacokinetic and hematologic toxicity data, significant correlations between the AUC of 9aminocamptothecin lactone and the percentage decrease in white blood cell count $(r=0.86 ; P<.001$; Figure $3, A$ ), the percentage decrease in platelet count $(r=0.83 ; P<.001$; Figure $3, B)$, and the percentage decrease in absolute neutrophil count $(r=0.66 ; P<$ .001 ; Figure $3, C$ ) could be shown. In addition, the worst observed myelotoxicity grade according to common toxicity criteria in the entire patient population correlated with the AUC of 9-aminocamptothecin lactone $(r=0.93 ; P<.001$; data not shown). The development of any myelotoxicity (grade 2 or worse) was associated with an AUC of 9-aminocamptothecin lactone $\geq 17.3 \mathrm{ng} \cdot \mathrm{h} / \mathrm{mL}$, using the Hill equation and data shown in Figure 2, $B$. Pharmacokinetic-pharmacodynamic relationships based on (log-)linear and nonsigmoidal $\mathrm{E}_{\max }$ models were less predictive, as were models based on 9-aminocamptothecin carboxylate or total 9-aminocamptothecin (data not shown).

\section{DISCUSSION}

Topoisomerase I inhibitors are of great clinical interest because of their unique mode of action, their important antitumor activity, and the high expression of the enzyme in various human tumor types. 9-Aminocamptothecin, a semisynthetic analog of camptothecin, revealed a broad antitumor activity in preclinical studies. Initial phase I studies focused on schedules with prolonged infusion duration. An oral formulation was developed to facilitate the prolonged drug administration. 9-Aminocamptothecin can be administered orally as a colloid dispersion or as gelatin capsules in PEG1000 . In dogs, the mean oral bioavailability of the colloid dispersion formulation was $13 \%$ (range, $4.5 \%$ to $26 \%$ ), compared with $10 \%$ of the PEG-1000. Both formulations retained their antitumor activity after oral administration. The phase I study on the oral administration of the colloid dispersion formulation of 9aminocamptothecin, 5 days per week every 2 weeks, 
was recently completed. ${ }^{25}$ Diarrhea was the doselimiting toxicity at a dose level of $0.2 \mathrm{mg} / \mathrm{m}^{2}$. 9-Aminocamptothecin with PEG-1000 has been shown previously to have rapid intestinal absorption in patients after oral delivery, with an overall bioavailability $(F)$ of $48.6 \pm 17.6 \%$. This compares favorably to other camptothecin analogs, including topotecan $(F=$ $30.0 \%),{ }^{26}$ 7-(4-methyl-piperazinomethylene)10,11-ethylenedioxy-20(S)-camptothecin (GI147211; $F=$ $11.3 \%),{ }^{27}$ and irinotecan $(F=12 \%$ to $21 \%) .{ }^{28}$ The terminal half-life of 9-aminocamptothecin was shown to be 12.6 to 4.20 hours, which is substantially longer than that of topotecan (range, 2.35 to 5.91 hours) ${ }^{26}$ and GI147211 (range, 6.85 to 3.13 hour). ${ }^{27}$ Another difference between topoisomerase I inhibitors constitutes the AUC ratio of the active lactone and the total drug of the parent drug. The conversion of 9-aminocamptothecin lactone into the ring-opened species in plasma could be shown from the first sample acquired (ie, at 0.33 hour). At equilibrium, the 9-aminocamptothecin carboxylate accounted for $91.1 \% \pm 2.11 \%$ of 9 -aminocamptothecin total drug concentrations.

This compares unfavorably to the percentages present in the ring-opened form at equilibrium observed in patients for topotecan $(\sim 50 \%),{ }^{26}$ GI147211 ( 60\%), ${ }^{27}$ and irinotecan and its active metabolite SN-38 ( $65 \%$ and $\sim 35 \%$, respectively). ${ }^{29}$ These figures underscore the important differences in pharmacokinetics between camptothecin analogs.

Our results of the phase I study with oral 9aminocamptothecin capsules indicated that the drug could be administered in a 14-day schedule repeated every 3 weeks with tolerable and manageable toxicity. ${ }^{30}$ The dose-limiting toxicities were a combination of thrombocytopenia plus neutropenia complicated by fever and diarrhea occurring at a dose level of 1.1 $\mathrm{mg} / \mathrm{m}^{2} /$ day. Other side effects were mild to moderate (common toxicity criteria grade 1 to 2 ) and consisted of nausea, vomiting, alopecia, mucositis, and fatigue. Although 9-aminocamptothecin demonstrated a linear pharmacokinetic behavior over the entire dose range studied, we observed that the AUC of 9-aminocamptothecin lactone was a better indicator for the observed hematologic toxicity than the dose. The intrapatient variability in AUC and peak drug levels was extremely small and averaged less than $10 \%$ for 9-aminocamptothecin lactone. However, the interpatient variability in the concentrations of 9-aminocamptothecin at each of the sample time points, as well as in the AUC was large, with values for the coefficient of variation as high as $99 \%$. In this study, the high variability in lactone to carboxylate interconversion was significantly related to individ-

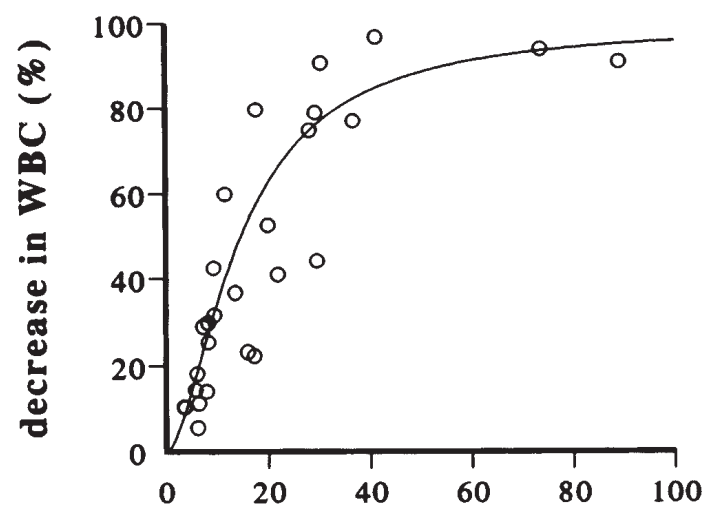

A

AUC 9-AC lac $(\mathrm{ng} . \mathrm{h} / \mathrm{mL})$

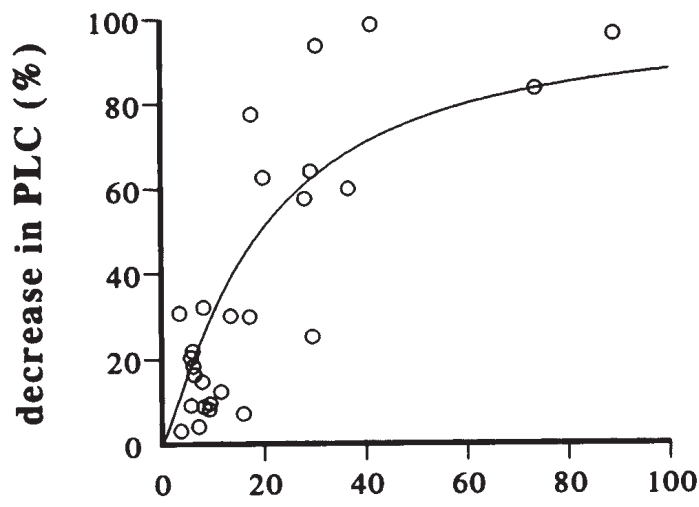

$B \quad A U C$ 9-AC lac $($ ng.h/mL)

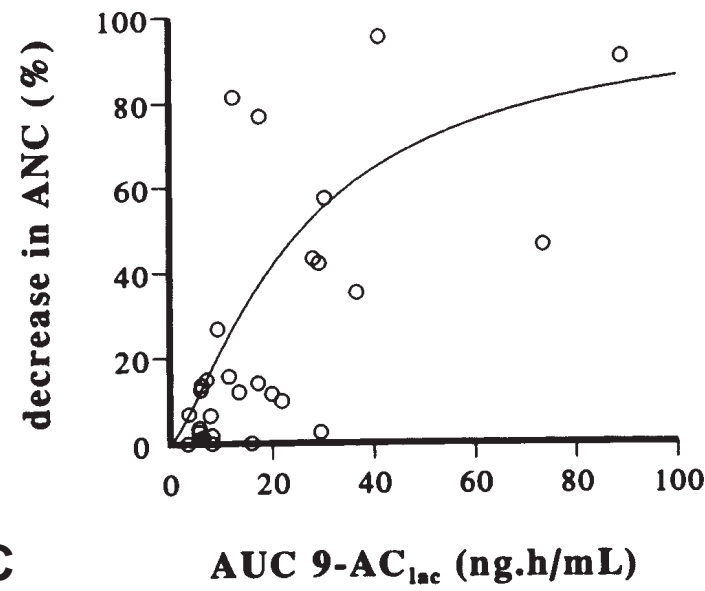

Figure 3. Correlation between the AUC of 9-aminocamptothecin lactone and the percentage decrease in white blood cells (WBC) at nadir of the first treatment course (A), in platelets (PLC; B) and in neutrophils (ANC; $\mathbf{C}$ ). The lines represent the fitting of the data to a sigmoidal maximumeffect model. 
ual differences in pretreatment serum albumin levels. Although our results need to be confirmed in a larger number of patients, they tend to indicate that higher protein levels will result in a more profound binding of 9aminocamptothecin carboxylate, thereby further diminishing the effective concentration of the active species of the drug. In all, these data indicate that classic drug dosing based on body surface area alone is unlikely to be effective in minimizing interpatient differences in systemic exposure to oral 9-aminocamptothecin.

The pharmacokinetics of 9-aminocamptothecin were clearly related to the pharmacodynamic outcome (ie, hematologic toxicity). The sigmoidal $\mathrm{E}_{\max }$ model was most appropriate to fit the kinetic data to the observed myelosuppression. The best correlation was obtained with the AUC of 9-aminocamptothecin lactone, the exposure to the active drug. Considering this pharmacokinetic-pharmacodynamic relationship, a target AUC for 9-aminocamptothecin lactone can be defined according to the grade of toxicity that is considered to be acceptable in future studies. If hematologic toxicity graded 2 or less is defined as acceptable, then the target AUC of 9-aminocamptothecin lactone is $17.3 \mathrm{ng} \cdot \mathrm{h} / \mathrm{mL}$, using the Hill equation and data shown in Figure 2, A.

For pharmacokinetic and pharmacodynamic analysis frequent blood sampling is inevitable. To evaluate salivary drug monitoring of 9-aminocamptothecin as an alternative to drug monitoring in plasma, the concentrations and AUC of 9-aminocamptothecin total and lactone were measured in unstimulated saliva samples in 5 patients during this study. The 9-aminocamptothecin concentration ratio in plasma and unstimulated saliva proved to be strongly patient-dependent and highly variable, suggesting that saliva is an unreliable matrix for pharmacokinetic analysis of 9-aminocamptothecin. Similar results were obtained recently for pharmacokinetic analysis of CPT-11 and SN-38 in saliva demonstrating large interpatient variability in plasma/saliva ratios. ${ }^{31}$

We recently developed a limited sampling model for reliable and accurate prediction of the systemic exposure to 9-aminocamptothecin after oral drug administration, using only a 1-time blood sample taken 3 hours after drug administration. ${ }^{32}$ To further diminish the interpatient variability in drug exposure in future studies, a pharmacokinetic-guided approach may be considered. After oral administration of an appropriate starting dose of 9-aminocamptothecin (ie, $1 \mathrm{mg} / \mathrm{m}^{2}$ ), the 9-aminocamptothecin lactone plasma concentration may then be measured at 3 hours after drug administration. With use of the limited sampling model and the linear-regression relationship between drug dose and
AUC (Figure 2), the optimal dose that leads to the target AUC (determined according to the toxicity considered to be acceptable) can be calculated.

This procedure may prove to be valuable in reducing interpatient variation in exposure to 9-aminocamptothecin, and it will enable us to optimize the treatment for any given patient by combining maximally achievable doses with tolerable toxicity during treatment. This strategy seems to be interesting in both additional phase II studies with the 14-day administration schedule and phase I studies with different schedules of administration. The clinical applicability of this concept is currently under investigation in our institute.

\section{References}

1. Wall ME, Wani MC, Cooke CE, Palmer KH, McPhail AT, Lim GA, et al. Plant antitumor agents; I: the isolation and structure of camptothecin, a novel alkaloidal leukemia and tumour inhibitor from Camptotheca acuminata. J Am Chem Soc 1966;88:3888-90.

2. Hsiang YH, Liu LF. Identification of mammalian DNA topoisomerase I as an intracellular target of the anticancer drug camptothecin. Cancer Res 1988;48:1722-6.

3. Eng WK, Faucette L, Johnson RK, Sternglanz R. Evidence that DNA topoisomerase I is necessary for the cytotoxic effects of camptothecin. Mol Pharmacol 1988;34:755-60.

4. Gottlieb JA, Guarino AM, Call JB, Oliverio VT, Block JB. Preliminary pharmacologic and clinical evaluation of camptothecin sodium (NSC 100880). Cancer Chemother Rep 1970;54:461-70.

5. Muggia FM, Creaven PJ, Hansen HH, Cohen MH, Selanrig OS. Phase I clinical trial of weekly and daily treatment with camptothecin (NSC 100880): correlation with preclinical studies. Cancer Chemother Rep 1972;56:515-21.

6. Moertel CCG, Schutt AJ, Reitemeier RJ, Hatin RG. Phase II study of camptothecin (NSC 100880) in the treatment of advanced gastrointestinal cancer. Cancer Chemother Rep 1972;56:95-101.

7. Fassberg J, Stella VJ. A kinetic and mechanistic study of the hydrolysis of camptothecin and some analogues. J Pharm Sci 1992;8:676-84.

8. Hertzberg RP, Caranfa MJ, Hecht SM. On the mechanism of topoisomerase I inhibition by camptothecin: evidence for binding to an enzyme-DNA complex. Biochemistry 1989;28:4629-38.

9. Takimoto CH, Klecker RW, Dahut W, Yee LK, Strong JM, Allegra CJ, et al. Analysis of the active lactone form of 9-aminocamptothecin in plasma using solid-phase extraction and high-performance liquid chromatography. J Chromatogr 1994;655:97-104.

10. Burke TG, Mi Z. The structural basis of camptothecin interactions with human serum albumin: impact on drug stability. J Med Chem 1994;37:40-6.

11. Pantazis P, Kozielski AJ, Vardeman DM, Petry ER, Giovanella BC. Efficacy of camptothecin congeners in the 
treatment of human breast carcinoma xenografts. Oncol Res 1993;5:273-81.

12. Giovanella BC, Stehlin JS, Hinz HR, Vardeman D, Mendoza JT, Potmesil M. Studies on time/dose intensity in treatment of human cancer xenografts with camptothecin analogues [abstract]. Proc Am Assoc Cancer Res 1994;35:455.

13. Pantazis P, Hinz HR, Mendoza JT, Kozielski AJ, Williams LJ, Stehlin JS, et al. Complete inhibition of growth followed by death of human malignant melanoma cells in vitro and regression of human melanoma xenografts in immunodeficient mice induced by camptothecins. Cancer Res 1992;52:3980-7.

14. De Souza PL, Cooper MR, Imondi AR, Myers CE. 9Aminocamptothecin: a topoisomerase I inhibitor with preclinical activity in prostate cancer. Clin Cancer Res 1997;3:287-94.

15. Supko JG, Malspeis L. Pharmacokinetics of the 9-amino and 10,11-methylenedioxy derivatives of camptothecin in mice. Cancer Res 1993;53:3062-9.

16. Supko JG, Plowman J, Dykes DJ, Zaharko DS. Relationship between the schedule dependence of 9-amino-20(S)camptothecin (AC; NSC603071) antitumor activity in mice and its plasma pharmacokinetics [abstract]. Proc Am Assoc Cancer Res 1992;33:432.

17. Rubin E, Wood V, Bharti A, Trites D, Lynch C, Hurwitz $\mathrm{S}$, et al. A phase I and pharmacokinetic study of a new camptothecin derivative, 9-aminocamptothecin. Clin Cancer Res 1995;1:269-76.

18. Dahut W, Harold N, Takimoto C, Allegra C, Chen A, Hamilton JM, et al. Phase I and pharmacologic study of 9-aminocamptothecin given by 72-hour infusion in adult cancer patients. J Clin Oncol 1996;14:1236-44.

19. Takimoto CH, Dahut W, Marino MT, Nakashima H, Liang MD, Harold N, et al. Pharmacokinetics and pharmacodynamics of a 72-hour infusion of 9-aminocamptothecin in adult cancer patients. J Clin Oncol 1997;15:1492-501.

20. Siu LL, Oza AM, Eisenhauer EA, Firby PS, Thiessen JJ, Michael M, et al. Phase I and pharmacologic study of 9aminocamptothecin colloidal dispersion formulation given as a 24-hour continuous infusion weekly times 4 every 5 weeks. J Clin Oncol 1998;16:1122-30.

21. Eder JP, Supko JG, Lynch T, Bryant M, Vosburgh E, Shulman LN, et al. Phase I trial of the colloidal dispersion formulation of 9-amino-20(S)-camptothecin administered as a 72-hour continuous intravenous infusion. Clin Cancer Res 1998;4:317-24.

22. Hochster H, Liebes L, Speyer J, Sorich J, Chachoua A,
Raphael B, et al. Phase I and pharmacodynamic study of prolonged infusion 9-amino-camptothecin (9AC) in two formulations [abstract] Proc Am Soc Clin Oncol 1997;16:201a.

23. Sparreboom A, De Jonge MJA, Punt CJA, Nooter K, Loos WJ, Porro MG, et al. Pharmacokinetics and bioavailability of oral 9-aminocamptothecin capsules in adult patients with solid tumors. Clin Cancer Res 1998;4:1915-9.

24. Loos WJ, Sparreboom A, Verweij J, Nooter K, Stoter G, Schellens JHM. Determination of the lactone and lactone plus carboxylate forms of 9-aminocamptothecin in human plasma by sensitive high-performance liquid chromatography with fluorescence detection. J Chromatogr B Biomed Sci Appl 1997;694:435-41.

25. Mani S, Iyer L, Janisch L, Wang X, Fleming GF, Schilsky RL, et al. Phase I clinical and pharmacokinetic study of oral 9-aminocamptothecin (NSC-603071). Cancer Chemother Pharmacol 1998;42:84-7.

26. Schellens JHM, Creemers GJ, Beijnen JH, Rosing H, De Boer-Dennert M, Davies B, et al. Bioavailability and pharmacokinetic of oral topotecan: a new topoisomerase I inhibitor. Br J Cancer 1996;73:1268-71.

27. Gerrits CJH, Schellens JHM, Creemers GJ, Wissel PS, Planting AST, Pritchard JF, et al. The bioavailability of oral GI147211 (GG211), a new topoisomerase I inhibitor. Br J Cancer 1997;76:946-51.

28. Stewart CF, Zamboni WC, Crom WR, Houghton PJ. Disposition of irinotecan and SN-38 following oral and intravenous irinotecan dosing in mice. Cancer Chemother Pharmacol 1997;40:259-65.

29. Rivory LP, Chatelut E, Canal P, Mathieu-Boué A, Robert J. Kinetics of the in vivo interconversion of the carboxylate and lactone forms of irinotecan (CPT-11) and of its metabolite SN-38 in patients. Cancer Res 1994;54:6330-3.

30. Punt CJA, de Jonge MJA, Sparreboom A, Loos WJ, van Beurden V, Planting AST, et al. Phase I and pharmacologic study on the topoisomerase I inhibitor [PEG-1000] 9-aminocamptothecin (9-AC) given orally to patients with solid rumors [abstract]. Proc Am Soc Clin Oncol 1998;17:197a.

31. Takahashi T, Fujiwara Y, Sumiyoshi H, Isobe T, Yamaoka N, Yamakido M. Salivary drug monitoring of irinotecan and its active metabolite in cancer patients. Cancer Chemother Pharmacol 1997;40:449-52.

32. Sparreboom A, de Jonge MJA, Nooter K, Loos WJ, Porro MG, Stoter G, et al. Prediction of the systemic exposure to oral 9-aminocamptothecin PEG1000 using limitedsampling models [abstract]. Proc Am Assoc Cancer Res 1998;39:363-4. 